University of Nebraska - Lincoln

DigitalCommons@University of Nebraska - Lincoln

Roger Kirby Publications

Research Papers in Physics and Astronomy

December 1984

\title{
Lattice Dynamics of Mixed Crystals of TiSe2
}

Roger D. Kirby

University of Nebraska-Lincoln, rkirby1@unl.edu

G.A. Freund, Jr.

University of Nebraska - Lincoln

Follow this and additional works at: https://digitalcommons.unl.edu/physics_kirby

Part of the Physics Commons

Kirby, Roger D. and Freund, Jr., G.A., "Lattice Dynamics of Mixed Crystals of TiSe2" (1984). Roger Kirby Publications. 24.

https://digitalcommons.unl.edu/physics_kirby/24

This Article is brought to you for free and open access by the Research Papers in Physics and Astronomy at DigitalCommons@University of Nebraska - Lincoln. It has been accepted for inclusion in Roger Kirby Publications by an authorized administrator of DigitalCommons@University of Nebraska - Lincoln. 


\title{
Lattice dynamics of mixed crystals of $\mathrm{TiSe}_{2}$
}

\author{
G. A. Freund, Jr.* and R. D. Kirby \\ Behlen Laboratory of Physics, University of Nebraska-Lincoln, Lincoln, Nebraska 68588
}

(Received 19 June 1984)

\begin{abstract}
We present the results of Raman scattering measurements on the mixed-crystal systems $\mathrm{TiSe}_{2-x} \mathrm{~S}_{x}, \mathrm{TiSe}_{2-x} \mathrm{Te}_{x}$, and $\mathrm{Zr}_{x} \mathrm{Ti}_{1-x} \mathrm{Se}_{2}$. At room temperature the pure compounds all have crystal structures represented by the $D_{3 d}^{3}(P \overline{3} m 1)$ space group with three atoms per unit cell. There are two Raman-active $\overrightarrow{\mathrm{k}}=\overrightarrow{0}$ phonons, one of $A_{1 g}$ symmetry and one of $E_{g}$ symmetry. In the $\mathrm{TiSe}_{2-x} \mathrm{Te}_{x}$ system, the $A_{1 g^{-}}$and $E_{g}$-phonon frequencies shift smoothly with concentration, and the shifts are well described by a simple virtual-crystal model. The other two systems exhibit a twomode behavior for which the virtual-crystal model does not correctly predict the observed frequency shifts. A random element isodisplacement model, based on Jaswal's model for the pure-crystal lattice dynamics of $\mathrm{TiSe}_{2}$, is developed. This model correctly predicts several features of the observed Raman spectra with no adjustable parameters.
\end{abstract}

\section{INTRODUCTION}

The layered-structure transition-metal dichalcogenides have been extensively investigated because a number of them undergo phase transitions to structurally distorted states at low temperatures. ${ }^{1-4}$ Since the phase transitions are to a large extent a result of the unusual Fermi-surface geometries of these compounds, most of the previous investigations have focused on their electronic properties. In particular, a considerable effort has gone into experiments in which the Fermi surface is modified through growth of doped or mixed crystals. ${ }^{1,5-12}$ In this paper, we also deal with mixed crystals of the layered compounds, but our primary concern is with their lattice-dynamical properties. The mixed-crystal systems investigated here include $\mathrm{TiSe}_{2-x} \mathrm{~S}_{x}, \mathrm{TiSe}_{2-x} \mathrm{Te}_{x}$, and $\mathrm{Zr}_{x} \mathrm{Ti}_{1-x} \mathrm{Se}_{2}$.

Single crystals of $\mathrm{TiSe}_{2}, \mathrm{TiS}_{2}, \mathrm{TiTe}_{2}$, and $\mathrm{ZrSe}_{2}$ all grow as the $1 T$ polytype, in which the metal ion is surrounded by six chalcogen ions in a nearly octahedral coordination. The space group for the structure is $D_{3 d}^{3}(P \overline{3} m 1)$, with one formula unit per crystalline unit cell. A grouptheoretical analysis predicts two Raman-active phonons, corresponding to the $A_{1 g}$ and $E_{g}$ irreducible representations of the $D_{3 d}$ point group, and two infrared-active phonons, of $A_{2 u}$ and $E_{u}$ symmetries. Raman and infrared measurements of the $\overrightarrow{\mathrm{k}}=\overrightarrow{0}$ phonons have been made on a number of $1 T$-structure layered compounds. ${ }^{13-20}$ Pure $\mathrm{TiSe}_{2}$ undergoes a phase transition to a structurally distorted state at $200 \mathrm{~K}$, in which the unit-cell dimensions increase to $2 a \times 2 a \times 2 c .^{21}$ This phase transition persists in the mixed crystals for substantial doping levels [up to $x \sim 0.95$ in $\operatorname{TiSe}_{2-x} S_{x} \quad$ (Ref. 6) and $x \sim 0.14$ in $\mathrm{Zr}_{x} \mathrm{Ti}_{1-x} \mathrm{Se}_{2}$ (Ref. 22)], and is responsible for the appearance of additional modes in the Raman spectra of these compounds. $\mathrm{TiSe}_{2}$ and $\mathrm{TiTe}_{2}$ are generally accepted to be small-band-overlap semimetals, ${ }^{23,24}$ whereas $\mathrm{TiS}_{2}$ and $\mathrm{ZrSe}_{2}$ are indirect-band-gap semiconductors with band gaps of $\sim 0.2$ and $\sim 1.2 \mathrm{eV}$, respectively. ${ }^{25,26}$ Thus one expects that the mixed crystals of $\mathrm{TiSe}_{2}$ with $\mathrm{TiS}_{2}$ and $\mathrm{ZrSe}_{2}$ will undergo a semimetal-semiconductor transfor- mation as $x$ is increased from 0 . More will be said about this later in connection with the lattice dynamics of these compounds.

The lattice dynamics of many mixed-crystal systems have been investigated both experimentally and theoretically, and several review articles have been published. ${ }^{27-29}$ A large fraction of the mixed-crystal systems thus far studied have been derived from cubic crystals with two atoms per unit cell and composition $A B_{1-x} C_{x}$, with most of the work being done on the alkali halides ${ }^{30}$ and the IIIV (Refs. 31 and 32) and II-IV (Refs. 33 and 34) compounds. Two main types of mixed-crystal phonon behavior have been observed. The term "one-mode" behavior refers to a system in which the phonon frequency changes monotonically with mixed-crystal composition, with the appearance of no additional modes. "Twomode" behavior refers to those cases where additional modes arising from impurity motion appear in the vicinity of the pure-crystal phonons. Some of these new modes remain fairly constant in frequency throughout the entire concentration range, while others are found to undergo considerable frequency shifts. These modes often become hard to trace at high mixing concentrations because of disorder-induced broadening.

Because of the difficulty of treating systems which do not have translational invariance, very few tractable models of mixed-crystal lattice dynamics have been developed. ${ }^{27-29}$ A number of numerical calculations on finite-model systems have been carried out, with much of this effort going into linear-chain models. Analytic models, including the virtual-crystal model, the more complicated isodisplacement models, and coherentpotential-approximation models have had some success in explaining the experimental results.

In this paper we present the results of Raman scattering measurements on the mixed crystals $\mathrm{TiSe}_{2-x} \mathrm{~S}_{x}$, $\mathrm{TiSe}_{2-x} \mathrm{Te}_{x}$, and $\mathrm{Zr}_{x} \mathrm{Ti}_{1-x} \mathrm{Se}_{x}$, and compare them with the predictions of the virtual-crystal model and the random element isodisplacement model. This latter model was developed by Verleur and Barker, ${ }^{31}$ and by Chen, 
Shockley, and Pearson, ${ }^{35}$ and it has enjoyed considerable success in describing the experimental results on several mixed-crystal systems, most notably $\mathrm{GaAs}_{1-x} \mathbf{P}_{x}$. In Sec. II we describe the growth and characterization of the crystals used in this investigation, and in Sec. III we present the Raman scattering data. These data show onemode behavior for $\mathrm{TiSe}_{2-x} \mathrm{Te}_{x}$ and two-mode behavior for $\mathrm{TiSe}_{2-x} \mathrm{~S}_{x}$ and $\mathrm{Zr}_{x} \mathrm{Ti}_{1-x} \mathrm{Se}_{2}$. In Sec. IV we describe a virtual-crystal model that quite accurately predicts the frequencies observed experimentally in $\mathrm{TiSe}_{2-x} \mathrm{Te}_{x}$ but not in the other two systems. Next, we recast the latticedynamical model of $\mathrm{Jaswal}^{36}$ for $\mathrm{TiSe}_{2}$ into the random element isodisplacement approximation. With this model we obtain considerable agreement with the experimental results for $\mathrm{TiSe}_{2-x} \mathrm{~S}_{x}$ and $\mathrm{Zr}_{x} \mathrm{Ti}_{1-x} \mathrm{Se}_{2}$. We end in Sec. $\mathrm{V}$ by speculating on certain features of the data and discussing the applicability of the random element isodisplacement model to systems of this sort.

\section{CRYSTAL GROWTH AND CHARACTERIZATION}

Mixed crystals of $\mathrm{TiSe}_{2-x} \mathrm{~S}_{x}, \mathrm{TiSe}_{2-x} \mathrm{Te}_{x}$, and $\mathrm{Zr}_{x} \mathrm{Ti}_{1-x} \mathrm{Se}_{2}$ were grown at $600^{\circ} \mathrm{C}$ using the vapor-phasetransport method with iodine as the transporting agent. Single crystals of $\mathrm{TiSe}_{2-x} \mathrm{~S}_{x}$ could be grown over the entire range of compositions $(0<x<2)$. In order to determine the compositions of these crystals, we made accurate lattice-constant measurements using both an $\mathrm{x}$-ray diffractometer and a Gandolfi rotating-crystal camera. The values of the $c$-axis lattice constants thus obtained agree with those of Rimmington and Balchin ${ }^{37}$ to within $0.2 \%$, with our measured values being consistently larger. This is to be expected since the relatively high growth temperatures used by Rimmington and Balchin should result in slightly metal-rich crystals, for which their data also show a decreasing $c$-lattice parameter as the amount of interstitial Ti increases. Thus we conclude that the actual compositions of our $\mathrm{TiSe}_{2-x} \mathrm{~S}_{x}$ crystals are very close to the nominal compositions. This conclusion is also confirmed by electrical-resistivity measurements, which were in good agreement with the results reported by DiSalvo, Moncton, and Waszczak. ${ }^{6}$ Single crystals of $\mathrm{TiSe}_{2-x} \mathrm{Te}_{x}$ could only be grown with values of $x$ near 0 or 2 . These crystals were not analyzed for Te concentration, and the values of $x$ assigned are the nominal values.

Single crystals of $\mathrm{Zr}_{x} \mathrm{Ti}_{1-x} \mathrm{Se}_{2}$ could only be grown for $x<0.21$, and the actual $\mathrm{Zr}$ concentrations were typically factors of 2-5 less than the intended concentrations, as determined by $\mathrm{x}$-ray fluorescence measurements. Thermopower measurements by Gaby et al. ${ }^{12}$ on crystals from the same growth batches showed a slight increase in the number of extrinsic carriers compared to pure $\mathrm{TiSe}_{2}$. Extended x-ray-absorption fine-structure (EXAFS) measurements by Davies and Brown, ${ }^{38}$ also on crystals from the same growth batches as those studied here, indicated that essentially all of the $\mathrm{Zr}$ substituted for Ti.

For Raman scattering measurements the samples were glued to a copper substrate using General Electric 7031 varnish and cleaved with Scotch Tape immediately prior to mounting in an exchange-gas liquid-nitrogen cryostat. Although the nominal temperatures were $80 \mathrm{~K}$, actual sample temperatures were typically $40-60 \mathrm{~K}$ higher due to local heating by the incident laser beam.

The Raman scattering apparatus consisted of a Coherent Radiation, Inc. model-52 argon-ion laser, a Spex model-1401 double monochromator, and photon-counting electronics. Instrumental resolution for the data shown was typically $4 \mathrm{~cm}^{-1}$. The Raman measurements were made employing a pseudo-Brewster-angle scattering geometry, with two cylindrical lenses (rotated by $90^{\circ}$ ) being used to focus the incident laser beam on the sample. ${ }^{39}$ The spectrometer was advanced in $0.5-\mathrm{cm}^{-1}$ increments and the data were recorded digitally using a Digital Equipment Corporation LSI-11 microcomputer system. Spectral calibration lines were included in each spectrum.

\section{EXPERIMENTAL RESULTS}

$$
\text { A. } \operatorname{TiSe}_{2-x} \mathbf{S}_{x}
$$

Figure 1 shows representative Raman spectra of the $\mathrm{TiSe}_{2-x} \mathrm{~S}_{x}$ mixed-crystal system. For these spectra the incident laser beam was polarized in the plane of incidence, and the scattered light was unanalyzed, except for $x=1.2$, where it was polarized in the plane of incidence. The

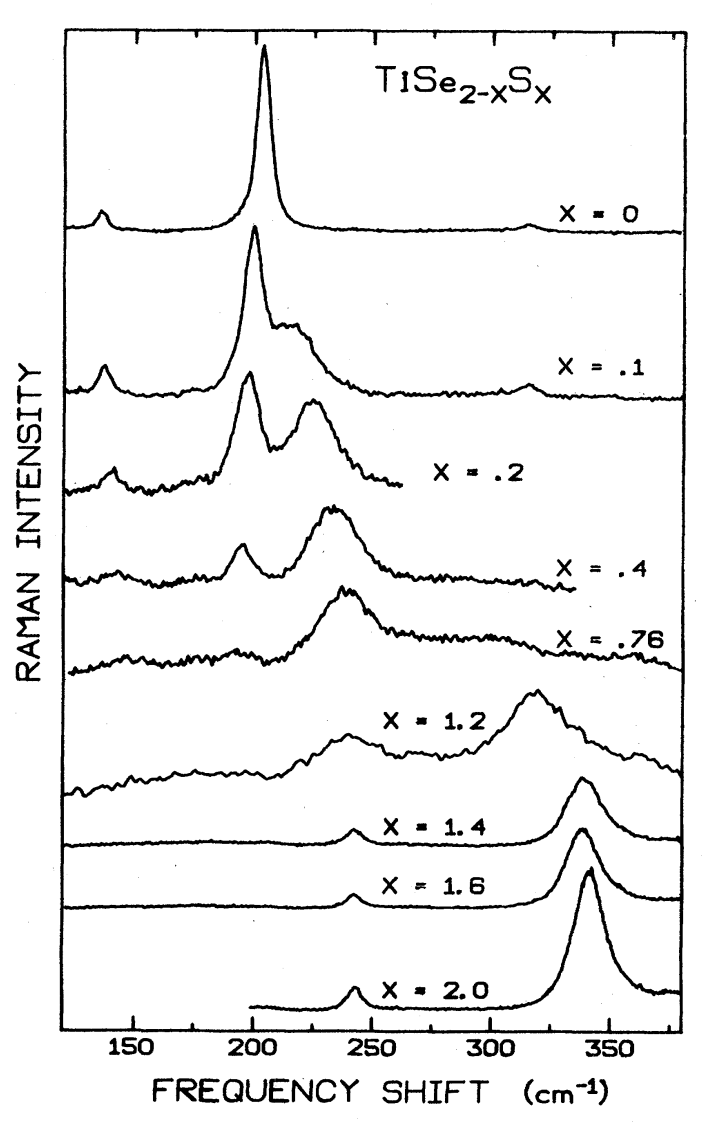

FIG. 1. Representative Raman spectra of the $\mathrm{TiSe}_{2-x} \mathrm{~S}_{x}$ mixed-crystal system $(x=0.0,0.1,0.2,0.4,0.76,1.2,1.4,1.6$, and 2.0). These data were obtained using the 488-nm argonlaser line and an instrumental resolution of $4 \mathrm{~cm}^{-1}$. The sample temperatures were approximately $125 \mathrm{~K}$. 


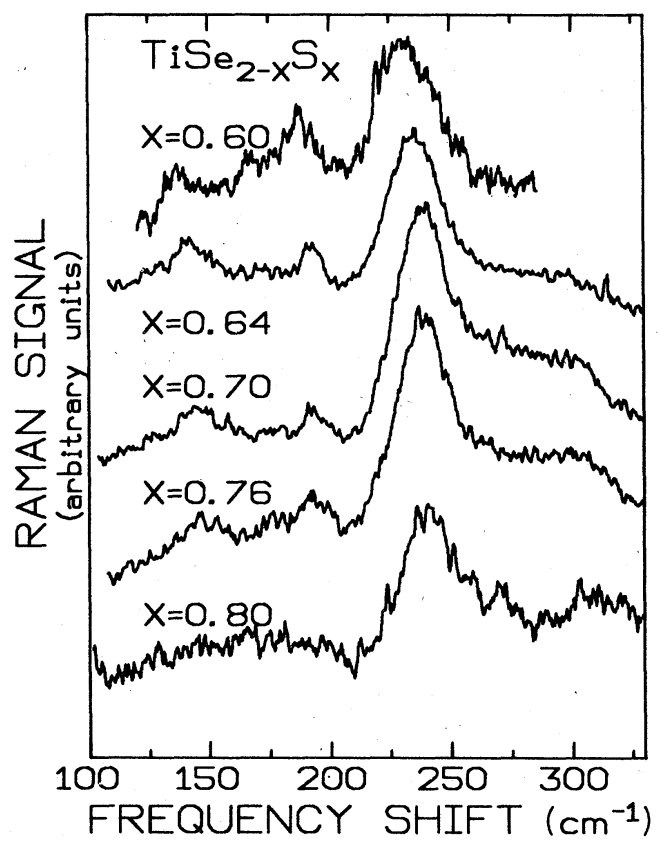

FIG. 2. Raman spectra of $\mathrm{TiSe}_{2-x} \mathrm{~S}_{x}$ for $0.6<x<0.8$. These data were obtained using the 488-nm argon-laser line and an instrumental resolution of $4 \mathrm{~cm}^{-1}$. The sample temperatures were approximately $125 \mathrm{~K}$.

symmetries of the various scattering peaks were determined in separate polarized Raman scattering measurements. The spectra of the pure crystals agree well with the results of Smith et al., ${ }^{13}$ Holy et al., ${ }^{16,40}$ and Sugai et $a l .{ }^{19}$ The sample temperatures, as determined by Stokes-anti-Stokes measurements, ranged from 134 to $155 \mathrm{~K}$. Note the behavior of the $A_{1 g}\left(204 \mathrm{~cm}^{-1}\right)$ and $E_{g}$ $\left(136 \mathrm{~cm}^{-1}\right)$ phonons as $x$ increases from zero. (Although it is not strictly correct to label the mixed-crystal phonons with the pure-crystal symmetry labels, we do so for ease of discussion.) At $x=0.1$, both the $A_{1 g}$ and $E_{g}$ peak heights are reduced and the widths are somewhat increased. In addition, an impurity-induced mode is clearly in evidence at $217 \mathrm{~cm}^{-1}$. The two-phonon peak at 316 $\mathrm{cm}^{-1}$ is still present in this scan. By $x=0.4$, the peak heights of all the modes are considerably reduced. By $x=0.76$, the $E_{g}$ mode has become so weak and broad that it is scarcely observable. It should also be noted that a weak and broad peak at approximately $310 \mathrm{~cm}^{-1}$ starts to appear in the $x=0.76$ spectrum. Between $x=0.6$ and 0.8 , the spectrum changes from $\mathrm{TiSe}_{2}$-like to $\mathrm{TiS}_{2}$-like. This will be discussed in more detail later. Polarization measurements indicate that both of the peaks appearing in the spectra for $x$ between 0.8 and 1.2 have $A_{1 g}$-like symmetry. As $x$ increases from 1.2 to 1.4 , the lowerfrequency mode $\left(242 \mathrm{~cm}^{-1}\right)$ changes from $A_{1 \mathrm{~g}}$-like to $E_{g}$-like symmetry. By $x=1.6$, the lines have sharpened considerably and a large two-phonon tail can be observed on the $A_{1 \mathrm{~g}}\left(338 \mathrm{~cm}^{-1}\right)$ peak. The peak heights also become successively larger with increasing sulfur content.

Figure 2 shows in detail the change in the character of the Raman spectra from the selenide-like to the sulfide-

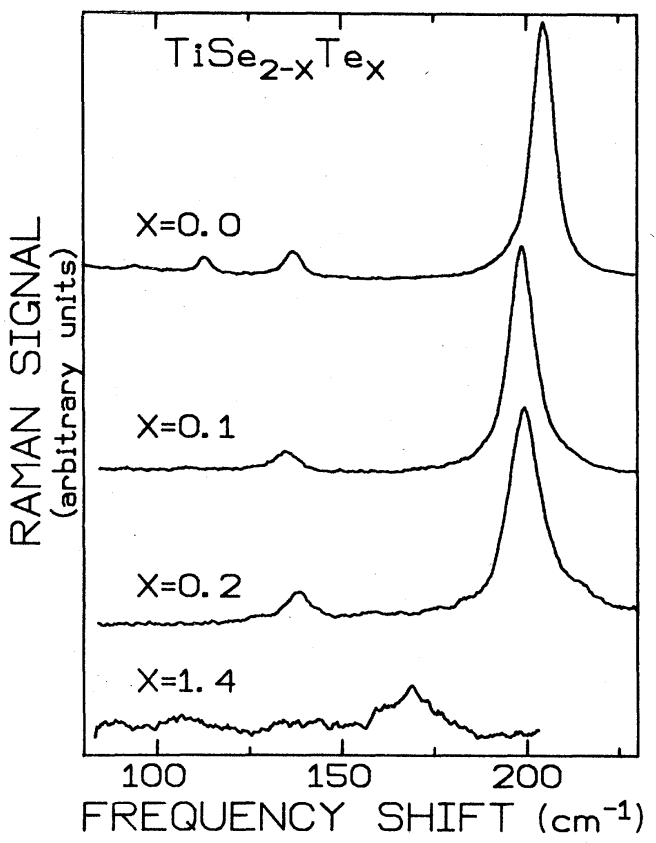

FIG. 3. Representative Raman spectra of the $\mathrm{TiSe}_{2-x} \mathrm{Te}_{x}$ mixed-crystal system $(x=0.0,0.1,0.2$, and 1.4). These data were obtained using the 488-nm argon-laser line and an instrumental resolution of $4 \mathrm{~cm}^{-1}$. The sample temperatures were approximately $125 \mathrm{~K}$.

like spectra for the region $100-330 \mathrm{~cm}^{-1}$. Notice that as $x$ increases from 0.6, the TiSe $A_{1 g}$ peak (at $195 \mathrm{~cm}^{-1}$ ) decreases in intensity and disappears by $x=0.8$, and a new peak, as is evident from Fig. 1, continues to evolve at larger $x$ to become the pure-TiS $A_{1 g}$ phonon.

\section{B. $\mathrm{TiSe}_{2-x} \mathrm{Te}_{x}$}

Figures 3 and 4 show the Raman spectra obtained from the $\mathrm{TiSe}_{2-x} \mathrm{Te}_{x}$ mixed-crystal system for nominal concentrations of $x=0.1,0.2,1.4,1.6,1.8$, and 2.0. First consider the spectrum of $\mathrm{TiTe}_{2}$, since it has not been previously reported in the literature. A Stokes-anti-Stokes determination of the temperature places it at $116 \mathrm{~K}$. Polarization-analyzed scans reveal that the 101- and 146$\mathrm{cm}^{-1}$ peaks have $E_{\mathrm{g}}$ and $A_{1 \mathrm{~g}}$ symmetries respectively. These peaks are quite narrow (the full width at half peak intensity is less than $4 \mathrm{~cm}^{-1}$ ), unlike the corresponding peaks in $\mathrm{TiSe}_{2}$ and $\mathrm{TiS}_{2}$. It seems reasonable to attribute the weak peak that is observed between the $E_{g}$ and $A_{1 g}$ peaks to two-phonon scattering because resistivity measurements show no anomaly indicative of superlattice formation.

As $x$ is decreased from 2.0, both the $A_{1 g}$ and the $E_{g}$ peaks increase in frequency and become very broad and weak by $x=1.6$. The weak two-phonon peak is also observable in these scans. For $x=0.2$ and 0.1 the spectra are very similar to the pure-selenide spectra above the transition temperature. The resistivity measurements of Kirby et al. ${ }^{22}$ show that the phase transition occurs at 


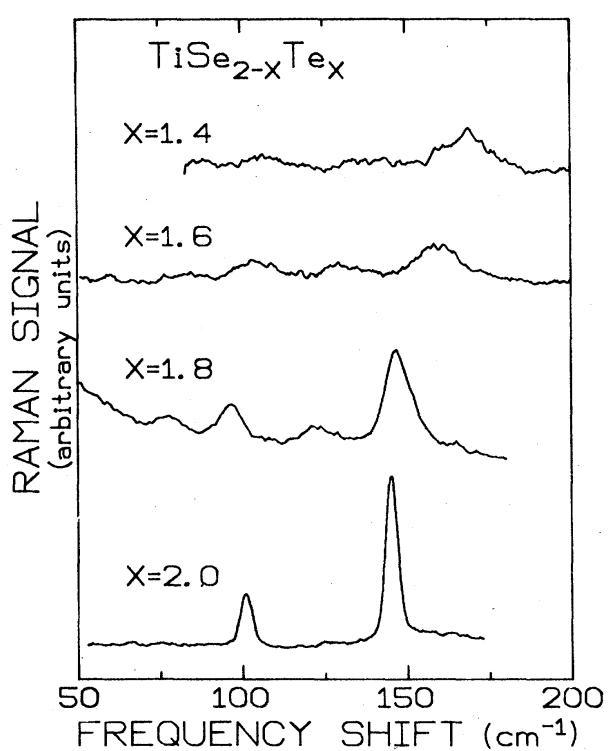

FIG. 4. Representative Raman spectra of the $\mathrm{TiSe}_{2-x} \mathrm{Te}_{x}$ mixed-crystal system $(x=1.4,1.6,1.8$, and 2.0$)$. These data were obtained using the 488-nm argon-laser line and an instrumental resolution of $4 \mathrm{~cm}^{-1}$. The sample temperatures were approximately $125 \mathrm{~K}$.

$115 \mathrm{~K}$ for the $x=0.1$ sample, but show no evidence for a phase transition in the $x=0.2$ sample. Thus no superlattice-induced peaks would be expected in these Raman spectra.

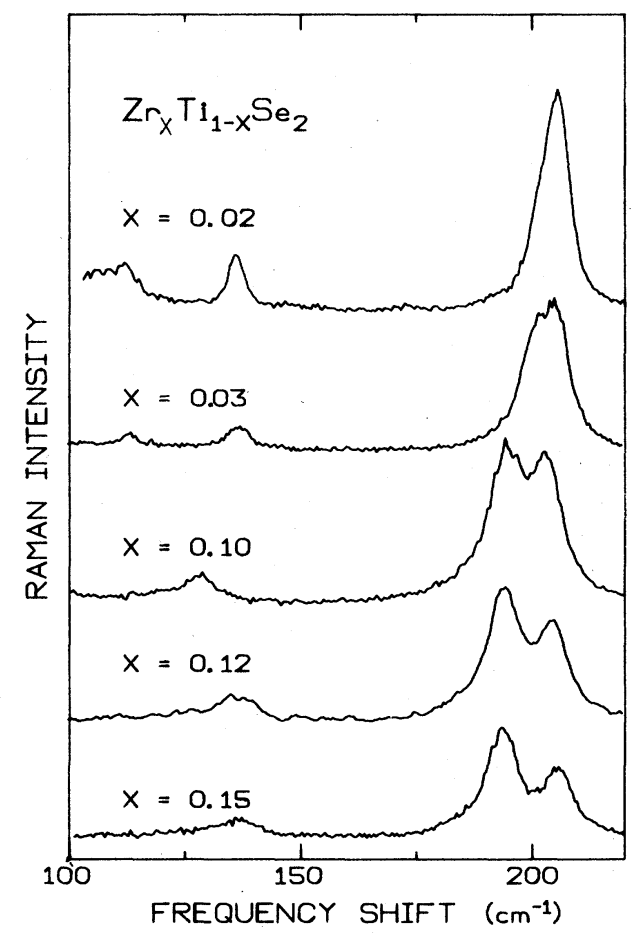

FIG. 5. Representative Raman spectra of the $\mathrm{Zr}_{x} \mathrm{Ti}_{1-x} \mathrm{Se}_{2}$ mixed-crystal system $(x=0.02,0.03,0.10,0.12$, and 0.15$)$. These data were obtained using the 488-nm argon-laser line and an instrumental resolution of $4 \mathrm{~cm}^{-1}$. The sample temperatures were approximately $125 \mathrm{~K}$.
C. $\mathrm{Zr}_{x} \mathrm{Ti}_{1-x} \mathrm{Se}_{2}$

Figure 5 shows the Raman spectra of $\mathrm{Zr}_{x} \mathrm{Ti}_{1-x} \mathrm{Se}_{2}$ for concentrations up to $x=0.21$. Sample temperatures determined by Stokes-anti-Stokes measurements were typically $110-120 \mathrm{~K}$. The $x=0.02$ spectrum shows the $E_{g}$ and $A_{1 g}$ phonons at 135 and $205 \mathrm{~cm}^{-1}$, respectively. The lower-frequency peaks are due to superlattice formation, and they will be discussed in a separate publication. Some broadening of the $A_{1 \mathrm{~g}}$ phonon is evident in the $x=0.03$ spectrum, and by $x=0.10$, two modes (both of $A_{1 g}$ symmetry) are clearly resolved. As $x$ is increased further, the lower-frequency mode shifts significantly downward in frequency, and the relative intensities of the two modes change markedly. The $E_{g}$ phonon shows very little frequency shift with increasing concentration.

\section{MODEL CALCULATIONS}

\section{A. $\operatorname{TiSe}_{2-x} \mathrm{Te}_{x}$}

Figure 6 shows the observed first-order Raman frequencies for the $\mathrm{TiSe}_{2-x} \mathrm{Te}_{x}$ mixed-crystal system as a function of $x$. The phonons of this system follow a onemode behavior which can be discussed in terms of a simple model. In the Raman-active modes of the pure crystals, the metal atoms occupy inversion sites and remain at rest while the chalcogenide atoms oscillate with equal amplitudes in opposite directions around these fixed points.

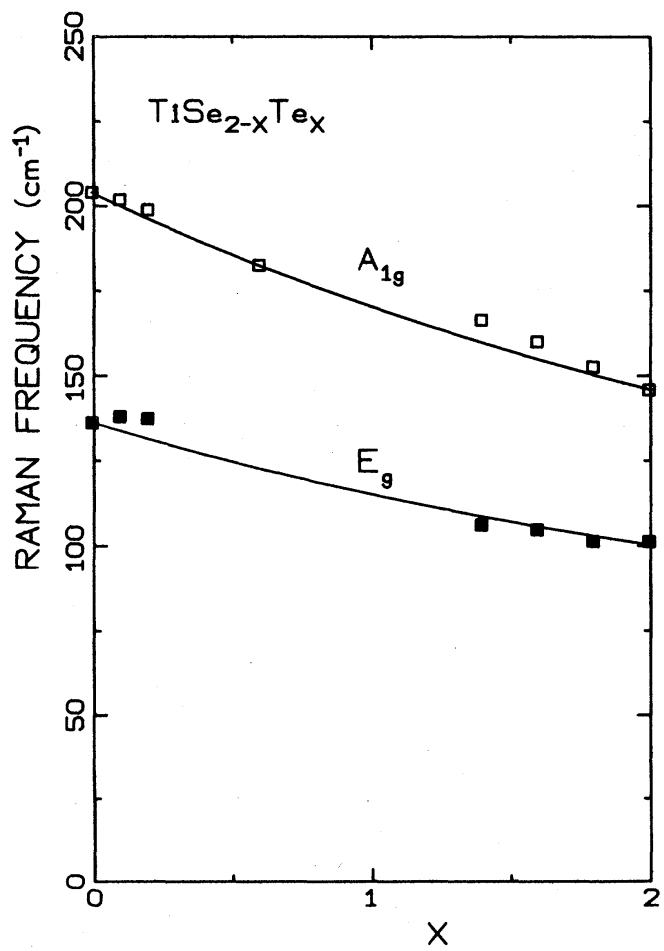

FIG. 6. Virtual-crystal calculation for the $A_{1 g^{-}}$and $E_{g^{-}}$ phonon frequencies in $\mathrm{TiSe}_{2-x} \mathrm{Te}_{x}$. The symbols are the observed frequencies and the solid curves are the calculated frequencies. 
Because the metal atoms remain at rest, the chalcogenides can be treated as simple harmonic oscillators whose frequency is given by

$$
\omega=\sqrt{K / M},
$$

where $K$ is the effective force constant and $M$ is the chalcogenide mass. The simplest approach to the lattice dynamics of mixed crystals is the virtual-crystal approximation, ${ }^{29}$ where the anion is replaced by an "average" anion whose mass and effective force constant depend linearly on the concentration. Thus, for $\mathrm{TiSe}_{2-x} \mathrm{Te}_{x}$, the mass and force constant in Eq. (1) become

$$
\begin{aligned}
& M=(1-x / 2) m_{0}+m_{2} x / 2, \\
& K=(1-x / 2) k_{0}+k_{2} x / 2,
\end{aligned}
$$

where $k_{0}$ and $k_{2}$ are the force constants and $m_{0}$ and $m_{2}$ are the chalcogenide masses for the pure $(x=0$ and 2$)$ compounds. The pure-crystal force constants $k_{A 0}, k_{A 2}$, $k_{E 0}$, and $k_{E 2}$ for the $A_{1 g}$ and $E_{g}$ modes can be determined from the observed Raman frequencies of the pure crystals. The results of these calculations are shown as the solid lines in Fig. 6. The agreement with experiment is quite good, with no adjustable parameters, indicating that more sophisticated calculations are not necessary. It should be noted that the force-constant changes between pure $\mathrm{TiSe}_{2}$ and pure $\mathrm{TiTe}_{2}$ amount to $12 \%$ for the $E_{g}$ mode and $21 \%$ for the $A_{1 g}$ mode.

$$
\text { B. } \operatorname{TiSe}_{2-x} \mathbf{S}_{x}
$$

\section{Virtual-crystal approximation}

There is little point in applying the virtual-crystal approximation to the $\mathrm{TiSe}_{2-x} \mathrm{~S}_{x}$ system, since it shows twomode behavior. However, it is interesting to note that many other studies of mixed crystals involving sulfideselenide combinations have revealed two-mode behavior. Examples of this are $\mathrm{ZnSe}_{1-x} \mathrm{~S}_{x},{ }^{33} \mathrm{CdSe}_{1-x} \mathrm{~S}_{x},{ }^{41}$ $\mathrm{HfSe}_{2-x} \mathrm{~S}_{x},{ }^{14}$ and amorphous $\mathrm{As}_{2} \mathrm{Se}_{3-x} \mathrm{~S}_{x} \cdot{ }^{42}$ Chang and Mitra $^{33}$ and Verleur and Barker ${ }^{31}$ have been somewhat successful in describing the behavior of the $\mathrm{ZnSe}_{1-x} \mathrm{~S}_{x}$ and $\mathrm{CdSe}_{1-x} \mathrm{~S}_{x}$ mixed-crystal systems using the random element isodisplacement model. It therefore seems reasonable to try such an approach for the $\mathrm{TiSe}_{2-x} \mathrm{~S}_{x}$ mixed crystals.

\section{Random element isodisplacement model}

The random element isodisplacement model was developed by Chen et al. ${ }^{35}$ to describe two-mode behavior in $\mathrm{GaAs}_{1-x} \mathbf{P}_{x}$ and other anion-doped mixed crystals of the form $A B_{1-x} C_{x}$. Here we describe the fundamental concepts involved in the random element isodisplacement (REI) model. In the zone-center optic phonon of GaAs and $\mathrm{GaP}$, the cation ( $\mathrm{Ga}$ ) sublattice beats against the anion (As or P) sublattice. In the REI model the anion sites throughout the crystal are assumed to be occupied randomly by one or the other of the two species of anions. Anions of like species are assumed to form rigid sublattices which vibrate as a unit. Thus, in this model, relative motion is possible only between atoms of different species-thus the reason for the term "isodisplacement." The random occupation of the anion sites is taken into account by averaging the effects of the impurities over all the unit cells of the pure structure. To do this, a new unit cell is considered in which the actual anion is relaced by a weighted "average anion," composed of $1-x$ of the mass of the species $B$ and $x$ of the mass of species $C$. The unit cell for the $A B_{1-x} C_{x}$ crystal is thus composed of three particles. By considering the average anion to be composed of two particles, an extra degree of freedom is introduced into the equations of motion. It is from this extra degree of freedom that the two-mode behavior arises. In the equations of motion the concentration factors multiply the anion masses and the anion-cation force constants. These features will be common to any lattice-dynamical model reformulated in the REI approximation.

The REI approximation can also be applied to mixed crystals of the transition-metal dichalcogenides. The crystal structure appropriate for the systems investigated here is shown in Fig. 7. The pure crystals of these compounds have three atoms per unit cell, with one cation and two anion sublattices before doping. One anion sublattice is composed of all the anions at sites equivalent to those indicated by an open circle in Fig. 7; the other sublattice is composed of all the anions at sites equivalent to those indicated by a half-filled circle. Thus, within the REI approximation, anion doping will introduce two additional

\section{(a)}
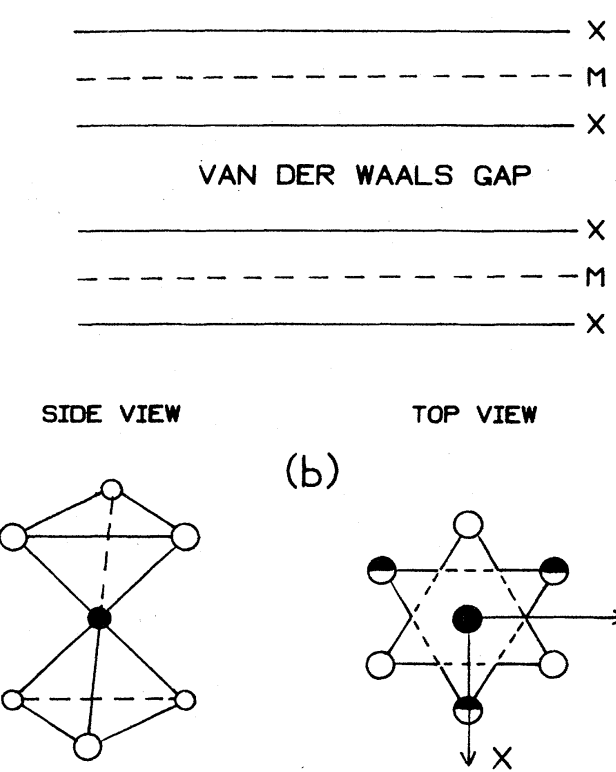

(b)
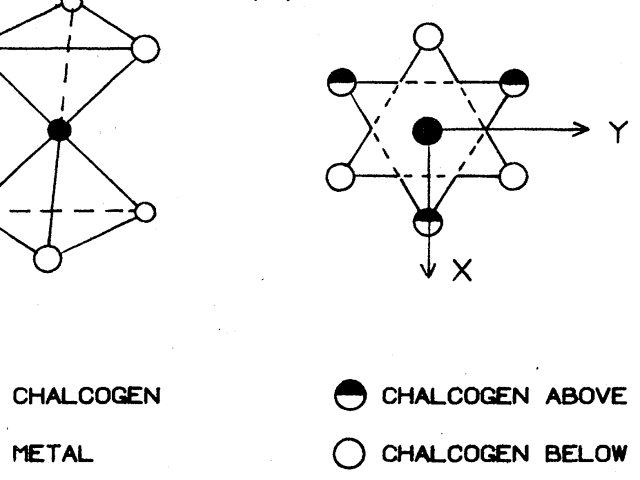

chalcogen above plane

CHALCOGEN BELOW PLANE

metal in plane

FIG. 7. Crystal structure of undistorted $\mathrm{TiSe}_{2}$. 
sublattices as described above.

Before the REI approximation can be applied, however, a good lattice-dynamical model is needed for the pure compounds. Jaswal ${ }^{36}$ has developed a short-range forceconstant model for the lattice dynamics of $\mathrm{TiSe}_{2}$. He calculated dispersion curves for all nine phonon branches and his results are in good agreement with the neutronscattering data reported by Wakabayashi et al. ${ }^{43}$ The simplicity of Jaswal's model makes it an attractive one to reformulate in the REI approximation and this is done here for the $\mathrm{TiSe}_{2-x} \mathrm{~S}_{x}$ system. Figure 8(a) shows the force constants involved in Jaswal's model for the zonecenter phonons.

The dynamical matrix for the $\vec{q}=\overrightarrow{0}$ pure-crystal phonons is $9 \times 9$ and, by symmetry, must take the form

$$
\left[\begin{array}{ccc}
\underline{A} & \underline{0}_{3 \times 3} & \underline{0}_{3 \times 3} \\
\underline{0}_{3 \times 3} & A & \underline{0}_{3 \times 3} \\
\underline{0}_{3 \times 3} & \underline{0}_{3 \times 3} & C
\end{array}\right],
$$

where $\underline{A}$ and $\underline{C}$ are themselves $3 \times 3$ matrices of the form

$$
\left[\begin{array}{ccc}
\frac{2 K}{M} & \frac{-K}{\sqrt{M m}} & \frac{-K}{\sqrt{M m}} \\
\frac{-K}{\sqrt{M m}} & \frac{K+k}{m} & \frac{-k}{m} \\
\frac{-K}{\sqrt{M m}} & \frac{-k}{m} & \frac{K+k}{m}
\end{array}\right] .
$$

Here, $M$ is the metal-ion mass and $m$ is the chalcogenide mass (see Fig. 8 for the force-constant notation).

Away from the zone center along the higher-symmetry directions, three of the transverse-phonon branches have displacements only in the $y$ direction, while the motion for the other six phonons couples the $x$ and $z$ displacements. Thus for $q \neq 0$, the form of the dynamical matrix and the matrices $A$ and $C$ is not the simple form indicated in Eqs. (4) and (5).

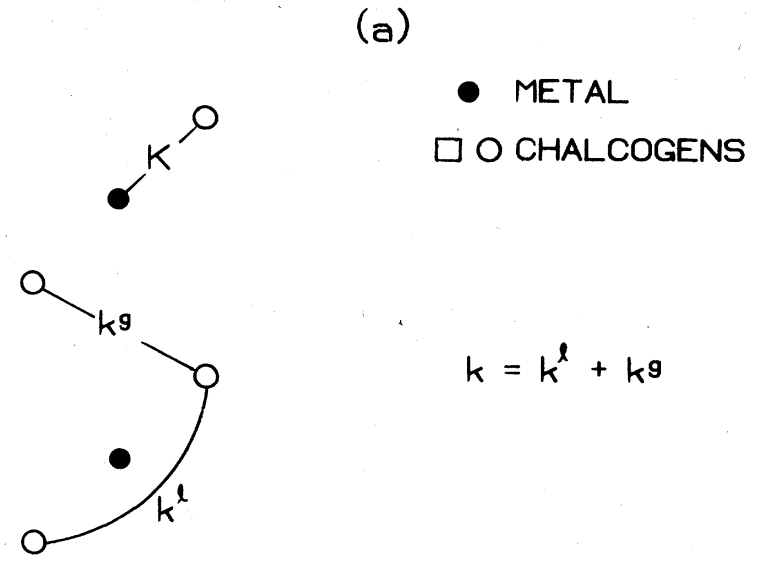

(b)

$$
\begin{aligned}
& k_{1}=k_{1}^{l}+k_{1}^{g} \\
& k_{2}=k_{2}^{l}+k_{2}^{g} \\
& k_{12}=k_{12}^{l}+k_{12}^{g}
\end{aligned}
$$

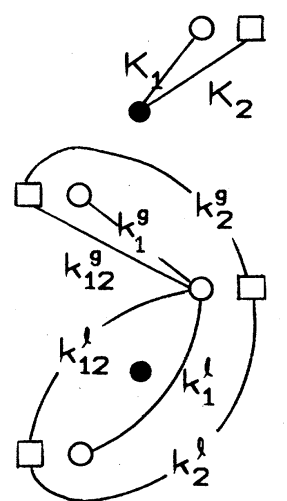

FIG. 8. (a) Short-range force constants in Jaswal's model of the lattice dynamics of $\mathrm{TiSe}_{2}$. (b) Force constants used in the random element isodisplacement adaptation of Jaswal's model.

When recast in the REI approximation, with appropriate concentration factors taken into account for both the masses and the force constants, $A$ and $C$ each become $5 \times 5$ matrices of the form

$$
\left[\begin{array}{ccccc}
\frac{2 K_{1}(1-x)+2 K_{2} x}{M} & \frac{-K_{1} \sqrt{1-x}}{\sqrt{M m_{1}}} & \frac{-K_{1} \sqrt{1-x}}{\sqrt{M m_{1}}} & \frac{-K_{2} \sqrt{x}}{\sqrt{M m_{2}}} & \frac{-K_{2} \sqrt{x}}{\sqrt{M m_{2}}} \\
\frac{-K_{1} \sqrt{1-x}}{\sqrt{M m_{1}}} & \frac{K_{1}+k_{1}(1-x)+k_{12} x}{m_{1}} & \frac{-k_{1}(1-x)}{m_{1}} & 0 & \frac{-k_{12} \sqrt{x(1-x)}}{\sqrt{m_{1} m_{2}}} \\
\frac{-K_{1} \sqrt{1-x}}{\sqrt{M m_{1}}} & \frac{-k_{1}(1-x)}{m_{1}} & \frac{K_{1}+k_{1}(1-x)+k_{12} x}{m_{1}} & \frac{-k_{12} \sqrt{x(1-x)}}{\sqrt{m_{1} m_{2}}} & 0 \\
\frac{-K_{2} \sqrt{x}}{\sqrt{M m_{2}}} & 0 & \frac{-k_{12} \sqrt{x(1-x)}}{\sqrt{m_{1} m_{2}}} & \frac{K_{2}+k_{2} x+k_{12}(1-x)}{m_{2}} & \frac{-k_{2} x}{m_{2}} \\
\frac{-K_{2} \sqrt{x}}{\sqrt{M m_{2}}} & \frac{-k_{12} \sqrt{x(1-x)}}{\sqrt{m_{1} m_{2}}} & 0 & \frac{-k_{2} x}{m_{2}} & \frac{K_{2}+k_{2} x+k_{12}(1-x)}{m_{2}}
\end{array}\right] .
$$

The matrices are $5 \times 5$ because in the random element isodisplacement model the anion doping introduces two additional particles per unit cell, which give rise to two additional equations of motion. Notice that if $x=0$, the
$3 \times 3$ matrix in the upper left-hand corner reduces to Eq. (5). The interaction term $k_{12}$ represents the interlayer and intralayer sulfur-selenium interactions. For simplicity in the calculations reported here it is taken to be 


$$
k_{12}=\left(k_{1}+k_{2}\right) / 2,
$$

that is, an arithmetic average of the selenium-selenium and sulfur-sulfur interlayer and intralayer force constants.

The only quantities needed to perform these calculations are the infrared and Raman frequencies of the two pure compounds, the atomic masses of the constituent atoms, and the concentration factors $x$ and $1-x$. The frequencies used in this calculation are listed in Table $I$. The $A_{2 u}$ modes are not well known because the samples are too thin parallel to the $c$ axis for normal-incidence reflectivity measurements to be carried out. Thus the $A_{2 u^{-}}$mode frequencies used in the calculations require some justification. In simple lattice-dynamical models developed by Bromley ${ }^{44}$ for $2 \mathrm{H}-\mathrm{MoS}_{2}$ and Duffey and $\mathrm{Kirby}^{39}$ for $1 T-\mathrm{TaS}_{2}$, the ratio of the $A_{2 u}$ to $E_{u}$ frequencies is approximately 1.22 . In Jaswal's model this ratio is not as simple to evaluate because it involves force constants which cannot be determined without knowing all the zone-center-mode frequencies. Since the $E_{u}$ mode in $\mathrm{TiSe}_{2}$ is known to be at $137 \mathrm{~cm}^{-1}$ in the normal state, multiplying this frequency by 1.22 would place the $A_{2 u^{-}}$ mode frequency at $167 \mathrm{~cm}^{-1}$. A shoulder at about 162 $\mathrm{cm}^{-1}$ appears on a large peak in the infrared data reported by Holy et al. ${ }^{16}$ This could be interpreted as the $A_{2 u}$ mode showing up in their spectra because of beam divergence. Wakabayashi et al. ${ }^{43}$ also show a zone-center mode in this region on their experimentally determined dispersion curves. Nothing is known about the $A_{2 u}$-mode frequency of $\mathrm{TiS}_{2}$, however. A value of $214 \mathrm{~cm}^{-1}$ (i.e., 1.22 times the $175-\mathrm{cm}^{-1} E_{u}$-mode frequency measured by Lucovsky et al. ${ }^{32}$ ) is used in this calculation. Once the phonon frequencies used in the calculation are determined, the model contains no adjustable parameters.

The eigenvalues and eigenvectors of the matrices [Eq. (6)] were determined using standard matrix-diagonalization techniques. Figure 9 shows the calculated $A_{1 g}$-like and $A_{2 u}$-like (solid curves) and $E_{g}$-like and $E_{u}$ like (dashed curves) phonon frequencies as a function of concentration. Of the five eigenvalues determined by the matrix diagonalization for each coordinate direction, one is always zero and corresponds to the zone-center acoustic phonon.

The highest-frequency mode shown in Fig. 9 starts out at low $x$ as an impurity mode. As $x$ increases this mode eventually becomes the pure-crystal $A_{1 \mathrm{~g}}$ mode of $\mathrm{TiS}_{2}$. The agreement between the calculated and observed frequencies is quite good in the range $0.60<x<2.0$. How-

TABLE I. Phonon frequencies $\left(\right.$ in $\mathrm{cm}^{-1}$ ) for the anion-doped mixed-crystal calculations.

\begin{tabular}{lcl}
\hline & $\mathrm{TiSe}_{2}$ & $\mathrm{TiS}_{2}$ \\
\hline$\omega\left(A_{1 g}\right)$ & 204 & 341 \\
$\omega\left(E_{g}\right)$ & 136 & 242 \\
$\omega\left(A_{2 u}\right)$ & $162^{\mathrm{a}}$ & $214^{\mathrm{a}}$ \\
$\omega\left(E_{u}\right)$ & $137^{\mathrm{b}}$ & $175^{\mathrm{c}}$ \\
\hline
\end{tabular}

${ }^{\mathrm{a} E s t i m a t e d ~ f r e q u e n c i e s . ~}$

${ }^{\text {b}}$ From Holy et al., Ref. 16.

${ }^{\mathrm{c}}$ From Lucovsky et al., Ref. 32.

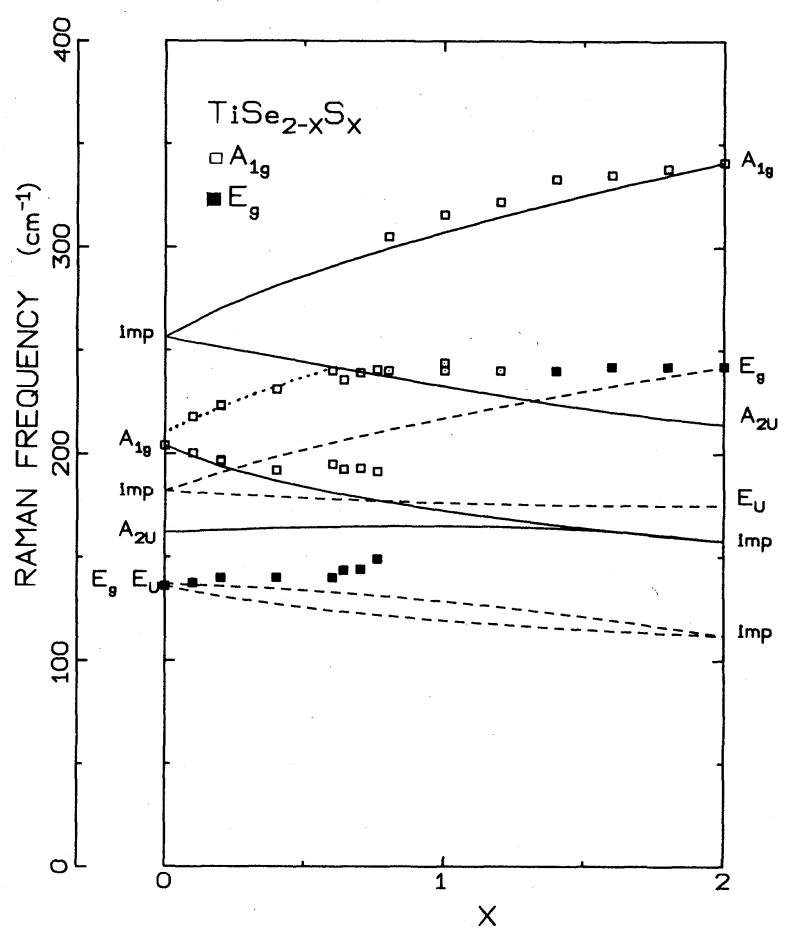

FIG. 9. Random element isodisplacement calculations for the phonons in $\mathrm{TiSe}_{2-x} \mathrm{~S}_{x}$. The open symbols are the measured $A_{1 g}$ frequencies, the solid symbols are the measured $E_{\mathrm{g}}$ frequencies, the solid curves are the calculated frequencies for the $A_{1 g}$ and $A_{2 u}$ phonons, and the dashed curves are the calculated frequencies for the $E_{g}$ and $E_{u}$ phonons. imp indicates an impurityinduced mode. The dotted curve is explained in the text.

ever, there is a large discrepancy for $0<x<0.60$. In this region, it is almost as if the calculated curve must be rigidly shifted down by $47 \mathrm{~cm}^{-1}$. The dotted curve in Fig. 9 is the upper curve shifted by this amount. Again the fit is quite good when this is done.

The lower-frequency $A_{1 g}$ mode, which originates at 204 $\mathrm{cm}^{-1}$ in pure $\mathrm{TiSe}_{2}$, and which decreases as $x$ increases, is expected to become the selenium impurity mode in $\mathrm{TiS}_{2}$. However, it cannot be observed for $x=0.30$. For $0<x<0.40$, the agreement between the calculated and the observed values is good, but becomes increasingly worse as $x$ increases to 0.80 . The reason for the disappearance of this mode is not understood, but it may be related to the unusual jump in frequencies noted for the higherfrequency $A_{1 g}$-like mode.

For the $E_{g}$-like and $E_{u}$-like modes, the agreement between the calculated and observed values is better than that found in the virtual-crystal calculation but certainly not as good as the agreement for the $A_{1 g}$-like modes. Between $x=0.6$ and 0.80 , the observed values show a gradual increase, but the mode cannot be observed for $x=0.80$. For $1.4<x<2.0$, polarization measurements indicate the mode at $240 \mathrm{~cm}^{-1}$ has $E_{\mathrm{g}}$-like character, but for $0.80<x<1.4$ the mode appearing at this frequency is observed to have $A_{1 \mathrm{~g}}$-like character. The apparent polarization change of this mode is not predicted by the random element isodisplacement model. Although this could be interpreted as a new mode appearing in the spectra, it is 
more likely a consequence of heavy doping relaxing the selection rules.

$$
\text { C. } \mathrm{Zr}_{x} \mathrm{Ti}_{1-x} \mathrm{Se}_{2}
$$

The $\mathrm{Zr}_{x} \mathrm{Ti}_{1-x} \mathrm{Se}_{2}$ family of mixed crystals shows a two-mode behavior for samples with $0.03<x<0.21$, which means that the virtual-crystal model will not agree with experiment. We have thus adapted Jaswal's model in the REI approximation in an attempt to explain the behavior of this system. Cation doping introduces only one additional degree of freedom because there is only one type of site involved in the substitution. The form of the matrices $A$ and $C$ in this case can thus be written as
TABLE II. Phonon frequencies (in $\mathrm{cm}^{-1}$ ) for the cationdoped mixed-crystal calculations.

\begin{tabular}{lcc}
\hline & $\mathrm{TiSe}_{2}$ & $\mathrm{ZrSe}_{2}$ \\
\hline$\omega\left(A_{1 g}\right)$ & 204 & $194^{\mathrm{a}}$ \\
$\omega\left(E_{g}\right)$ & 136 & $148^{\mathrm{a}}$ \\
$\omega\left(A_{2 u}\right)$ & $162^{\mathrm{b}}$ & $124^{\mathrm{b}}$ \\
$\omega\left(E_{u}\right)$ & $137^{\mathrm{c}}$ & $102^{\mathrm{d}}$ \\
\hline
\end{tabular}

${ }^{a}$ From Smith et al., Ref. 13.

${ }^{b}$ Estimated frequencies.

'From Holy et al., Ref. 16.

${ }^{\mathrm{d}}$ From Ratajack et al., Ref. 45.

$$
\left[\begin{array}{cccc}
\frac{2 K_{2}}{M_{2}} & 0 & \frac{-K_{2} \sqrt{x}}{\sqrt{M_{2} m}} & \frac{-K_{2} \sqrt{x}}{\sqrt{M_{2} m}} \\
0 & \frac{2 K_{1}}{M_{1}} & \frac{-K_{1} \sqrt{1-x}}{\sqrt{M_{1} m}} & \frac{-K_{1} \sqrt{1-x}}{\sqrt{M_{1} m}} \\
\frac{-K_{2} \sqrt{x}}{\sqrt{M_{2} m}} & \frac{-K_{1} \sqrt{1-x}}{\sqrt{M_{1} m}} & \frac{K_{1}(1-x)+K_{2} x+k_{1}}{m} & \frac{-k_{1}}{m} \\
\frac{-K_{2} \sqrt{x}}{\sqrt{M_{2} m}} & \frac{-K_{1} \sqrt{1-x}}{\sqrt{M_{1} m}} & \frac{-k_{1}}{m} & \frac{K_{1}(1-x)+K_{2} x+k_{1}}{m}
\end{array}\right] \text {, }
$$

where $M_{1}$ and $M_{2}$ are the cation masses and $m$ is the chalcogen mass. Again notice that, if $x=0$, the $3 \times 3$ matrix in the lower right-hand corner reduces to Eq. (5).

The calculations are performed in a manner similar to those for anion doping. The results of these calculations are shown in Fig. 10 for the $A_{1 g}$-like and $A_{2 u}$-like modes (solid curves) and the $E_{g}$-like and $E_{u}$-like modes (dashed curves). Table II lists the pure-crystal phonon frequencies used as input parameters for these calculations. Again, the $A_{2 u}$-mode frequencies are estimated as in the aniondoped case. $\mathrm{The} \mathrm{Zr}_{\boldsymbol{x}} \mathrm{Ti}_{1-x} \mathrm{Se}_{2}$ mixed-crystal system qualitatively behaves much the same as the $\mathrm{TiSe}_{2-x} \mathrm{~S}_{x}$ system. The room-temperature Raman data of Smith et al. ${ }^{13}$ reveal that the $A_{1 g}$ modes of $\mathrm{TiSe}_{2}$ and $\mathrm{ZrSe}_{2}$ differ by only $1 \mathrm{~cm}^{-1}$, while the $E_{g}$ modes differ by $12 \mathrm{~cm}^{-1}$. The observed modes at $204 \mathrm{~cm}^{-1}\left(A_{1 \mathrm{~g}}\right)$ and $136 \mathrm{~cm}^{-1}\left(E_{g}\right)$ in the $\mathrm{Zr}_{\boldsymbol{x}} \mathrm{Ti}_{1-x} \mathrm{Se}_{2}$ mixed-crystal system are similar to those of pure $\mathrm{TiSe}_{2}$ up to $x=0.21$, and the agreement between the observed frequencies and those calculated in the REI approximation for these modes is excellent. The additional $A_{1 g}$ mode observed in the spectra is an impurity mode. The calculation predicts two impurity modes at frequencies considerably lower than those observed.

\section{DISCUSSION}

FIG. 10. Random element isodisplacement calculations for the phonons in $\mathrm{Zr}_{x} \mathrm{Ti}_{1-x} \mathrm{Se}_{2}$. The open symbols are the measured $A_{1 g}$ frequencies, the solid symbols are the measured $E_{g}$ frequencies, the solid curves are the calculated frequencies for the $A_{1 g}$ and $A_{2 u}$ phonons, and the dashed curves are the calculated frequencies for the $E_{g}$ and $E_{u}$ phonons. imp indicates an impurity-induced mode.

\section{A. $\mathrm{TiSe}_{2-x} \mathbf{S}_{x}$}

As was discussed earlier and as can be clearly seen from the experimental data points in Fig. 9, the Raman spectrum of $\mathrm{TiSe}_{2-x} \mathrm{~S}_{x}$ changes abruptly in the vicinity of $x=0.80$. This is attributed to concentration-induced 
changes in the electronic band structure. Band-structure calculations by Zunger and Freeman ${ }^{23}$ indicate that $\mathrm{TiS}_{2}$ is an indirect-gap semiconductor $\left(E_{g}=0.23 \mathrm{eV}\right)$ and that $\mathrm{TiSe}_{2}$ is an indirect-overlap semimetal $\left(E_{g}=-0.18 \mathrm{eV}\right)$. These calculations have been found to be in good agreement with the experimental optical and electronic data. In view of this, $\mathrm{TiSe}_{2-x} \mathrm{~S}_{x}$ must, for some value of $x$, transform from a semimetal to a semiconductor as $x$ is increased from zero. If the bands are assumed to shift rigidly with concentration, the energy gap can be approximated by

$$
E_{g}(x)=(0.205 x-0.18) \mathrm{eV},
$$

which predicts that $E_{g}(0.88)=0$.

There are other data to support this view. A careful look at the lattice constants measured by Rimmington and Balchin $^{37}$ for the $\mathrm{TiSe}_{2-x} \mathrm{~S}_{x}$ mixed crystals reveals a kink (i.e., a change in the slope) in the lattice constants plotted as functions of concentration near $x=0.80$. This may be an indication of the semimetal-semiconductor transformation. The resistivity data of DiSalvo and Waszczak ${ }^{7}$ show that the anomaly indicative of superlattice formation disappears at $x=0.95 \pm 0.05$. This is also close to the estimate of the concentration at which the semimetal-tosemiconductor transformation is expected to occur. The observed resistivities for $1.0<x<2.0$ are metalliclike. Wilson ${ }^{46}$ has attributed such behavior in pure $\mathrm{TiS}_{2}$ to excess free carriers, introduced by nonstoichiometry, occupying the lowest conduction band. A similar behavior may be expected for the mixed crystals with $x=1.0$.

The discontinuous changes in the Raman frequencies near $x=0.80$ may result from the semimetal-tosemiconductor transformation. This is not likely to be due to a loss of screening electrons at the semimetal-tosemiconductor transformation because excess electrons are expected to be introduced by nonstoichiometry. However, the discontinuous change in the frequencies may mean that the screening length is smaller in the semimetal than in the semiconductor, although it can still be considered short range in both phases. Sulfur is certainly more electronegative than selenium, and the clustering of the sulfur atoms around the metal atoms and next to the selenium atoms at high concentrations may be responsible for a decrease in the screening. The bond angles determined from structure-refinement data reveal that $\mathrm{TiS}_{2}$ has a structure that is closer to the ideal octahedral structure than that of $\mathrm{TiSe}_{2}{ }^{47,48}$ This implies that subtle changes in the bonding or orbital hybridization might also be expected.

$$
\text { B. } \mathrm{Zr}_{x} \mathrm{Ti}_{1-x} \mathrm{Se}_{2}
$$

$\mathrm{ZrSe}_{2}$ is an indirect-band-gap semiconductor $\left(E_{g}=1.2\right.$ $\mathrm{eV}$ ), and, in the rigid-band approximation, the energy gap of $\mathrm{Zr}_{x} \mathrm{Ti}_{1-x} \mathrm{Se}_{2}$ as a function of $x$ can be expressed as

$$
E_{g}(x)=(1.38 x-0.18) \mathrm{eV} \text {. }
$$

This predicts $E_{g}(0.13)=0$. Resistivity ${ }^{22}$ and thermopower ${ }^{12}$ measurements reveal no anomaly indicative of superlattice formation in samples with $0.15<x<0.21$ at temperatures above $4.2 \mathrm{~K}$. Since a similar behavior has been observed in the $\mathrm{TiSe}_{2-x} \mathrm{~S}_{x}$ system near the semimetal-to-semiconductor transformation, ${ }^{6}$ this may indicate that the semimetal-to-semiconductor transformation and the suppression of the superlattice are related.

\section{Comments on the random element isodisplacement model}

The random element isodisplacement model used here has several drawbacks. First, this model forces the crystal to be translationally invariant, with a definite unit cell. This, of course, is unrealistic. Second, the effects of doping at low concentrations are not expected to be accurately described within this model because it fails to properly take into account local changes in the force constants. For extremely low concentrations, the impurity mode is expected to be a localized mode whose frequency need not be close to any of those of the host lattice, unlike the frequencies calculated in the random element isodisplacement calculation. Only at larger concentrations should the curves representing the frequencies calculated in the random element isodisplacement model and the frequencies of the localized modes become identical. In addition, at low concentrations the mass and force-constant changes are highly localized at the impurity sites, and it is not valid to average the effects of the doping over all the unit cells of the crystal as is done in this model.

In the case of $\mathrm{TiSe}_{2-x} \mathrm{~S}_{x}$ the random element isodisplacement model cannot be expected to describe the discontinuous behavior of the phonon frequencies at the semimetal-to-semiconductor transition. The model assumes that the masses and force constants change monotonically and continuously as the crystal composition is varied between the two pure compounds.

In spite of the objectional features, for most of the modes the agreement between the calculated and observed frequencies is good, especially considering that there are no adjustable parameters in the model. Although there are still many gaps in the understanding of the lattice dynamics of these materials, the calculations presented here have been useful in providing a framework within which to discuss the data and in pointing out some of the unusual features of mixed crystals of $\mathrm{TiSe}_{2}$.

\section{ACKNOWLEDGMENTS}

We wish to thank S. S. Jaswal for many helpful discussions regarding the lattice dynamics of both pure and mixed crystals. This research was supported by the $\mathrm{Na}$ tional Science Foundation under Grants No. DMR-7710217 and No. DMR-79-10025. 
*Present address: Boeing Military Aircraft Company, P.O. Box 7730, Wichita, KS 67277-7730.

1J. A. Wilson, F. J. DiSalvo, and S. Mahajan, Adv. Phys. 24, 117 (1975).

${ }^{2}$ C. B. Scruby, P. M. Williams, and G. S. Parry, Philos. Mag. 31, 255 (1975).

${ }^{3}$ K. C. Woo, F. C. Brown, W. L. McMillan, R. J. Miller, M. J. Schaffman, and M. P. Sears, Phys. Rev. B 14, 3242 (1976).

${ }^{4}$ F. R. Shepherd and P. M. Williams, J. Phys. C 7, 4416 (1974); 7, 4427 (1974).

5F. J. DiSalvo, J. A. Wilson, and J. V. Waszczak, Phys. Rev. Lett. 36, 885 (1976).

${ }^{6}$ F. J. DiSalvo, D. E. Moncton, and J. V. Waszczak, Phys. Rev. B 14, 4321 (1976).

${ }^{7}$ F. J. DiSalvo and J. V. Waszczak, Phys. Rev. B 17, 3801 (1978).

${ }^{8}$ W. Y. Liang, G. Lucovsky, R. M. White, and W. Stutius, Philos. Mag. 33, 493 (1976).

${ }^{9}$ F. Levy and Y. Froidevaux, J. Phys. C 12, 473 (1979).

${ }^{10}$ F. Levy, J. Phys. C 13, 2901 (1980).

${ }^{11}$ I. Taguchi, Solid State Commun. 32, 679 (1979).

12J. H. Gaby, B. DeLong, F. C. Brown, R. D. Kirby, and F. Levy, Solid State Commun. 39, 1167 (1981).

13J. E. Smith, M. I. Nathan, M. W. Shafer, and J. B. Torrance, in Proceedings of 11th International Conference of Physics in Semiconductors, Warsaw, Poland, 1972 (Elsevier, New York, 1972), p. 1306.

${ }^{14}$ G. Lucovsky, R. M. White, J. A. Benda, and J. F. Revelli, Phys. Rev. B 7, 3859 (1973).

15J. R. Duffey, R. D. Kirby, and R. V. Coleman, Solid State Commun. 20, 617 (1976).

16J. A. Holy, K. C. Woo, M. V. Klein, and F. C. Brown, Phys. Rev. B 16, 3628 (1977).

${ }^{17}$ D. Walsh, S. Jandl, and J. Y. Harbec, J. Phys. C 13, L125 (1980).

${ }^{18}$ D. R. Karecki and B. P. Clayman, Solid State Commun. 19, 479 (1976); Phys. Rev. B 19, 6367 (1979).

${ }^{19}$ S. Sugai, K. Murase, S. Uchida, and S. Tanaka, Solid State Commun. 35, 433 (1980).

20J. A. Wilson, A. S. Barker, F. J. DiSalvo, and J. A. Ditzenberger, Phys. Rev. B 18, 2866 (1978).
${ }^{21}$ K. C. Woo, F. C. Brown, W. L. McMillan, R. J. Miller, M. J. Schaffman, and M. P. Sears, Phys. Rev. B 14, 3242 (1976).

${ }^{22}$ R. D. Kirby, R. F. Fagerquist, and W. R. Nieveen, Solid State Commun. 51, 131 (1984).

${ }^{23}$ A. Zunger and A. J. Freeman, Phys. Rev. B 17, 1839 (1978).

${ }^{24}$ D. W. Bullett, J. Phys. C 12, 4501 (1978).

${ }^{25}$ E. M. Logothetis, W. J. Kaiser, C. A. Kukkonen, S. P. Faile, R. Colella, and J. Gambold, J. Phys. C 12, 6521 (1979).

${ }^{26}$ D. L. Greenaway and R. Nitsche, J. Phys. Chem. Solids 26, 1445 (1965).

${ }^{27}$ I. F. Chang and S. S. Mitra, Adv. Phys. 20, 359 (1971).

${ }^{28}$ R. J. Elliot, J. A. Krumhansl, and P. L. Leath, Rev. Mod. Phys. 46, 465 (1974).

${ }^{29}$ A. S. Barker and A. J. Sievers, Rev. Mod. Phys. 47, Suppl. 2, S1 (1975).

30I. R. Nair and C. T. Walker, Phys. Rev. B 7, 2740 (1973).

${ }^{31}$ H. W. Verleur and A. S. Barker, Phys. Rev. 149, 715 (1966).

${ }^{32}$ G. Lucovsky and M. F. Chen, Solid State Commun. 8, 1397 (1970).

33I. F. Chang and S. S. Mitra, Phys. Rev. 172, 924 (1968).

${ }^{34}$ R. Beserman and M. Balkanski, Phys. Rev. B 1, 608 (1970).

${ }^{35}$ Y. S. Chen, W. Shockley, and G. L. Pearson, Phys. Rev. 151, 648 (1966).

36S. S. Jaswal, Phys. Rev. B 20, 5297 (1979).

${ }^{37}$ H. B. R. Rimmington and A. A. Balchin, J. Cryst. Growth 21, 171 (1974).

${ }^{38}$ B. M. Davies and F. C. Brown, Phys. Rev. B 25, 2997 (1982).

39J. R. Duffey and R. D. Kirby, Phys. Rev. B 23, 1534 (1981).

40J. A. Holy, M. V. Klein, W. L. McMillan, and S. F. Meyer, Phys. Rev. Lett. 37, 1145 (1976).

${ }^{41}$ H. W. Verleur and A. S. Barker, Phys. Rev. 155, 750 (1967).

42R. Zallen and M. Slade, Phys. Rev. B 9, 1627 (1974).

${ }^{43}$ H. Wakabayashi, H. G. Smith, K. C. Woo, and F. C. Brown, Solid State Commun. 28, 923 (1978).

${ }^{44}$ R. A. Bromley, Philos. Mag. 23, 1417 (1971).

${ }^{45}$ M. J. Ratajack, C. R. Kannewurf, J. F. Revelli, and J. B. Wagner, Phys. Rev. B 17, 4674 (1978).

46J. A. Wilson, Phys. Status Solidi B 86, 11 (1978).

${ }^{47}$ A. H. Thompson, F. R. Gamble, and C. R. Symon, Mater. Res. Bull. 10, 915 (1975).

${ }^{48}$ C. Riekel, J. Solid State Chem. 17, 389 (1976). 